\title{
Avaliação de metodologias para a detecção de cepas de Staphylococcus aureus resistentes à meticilina (MRSA) e análise do perfil de sensibilidade frente aos antimicrobianos em um hospital terciário
}

\author{
Liliana Urdangarin de Sousa*, Tassiane Paz Mielke*, Rosmari \\ Horner ${ }^{\star * *}$, Mônica de Abreu Rodrigues**, Silvana Oliveira dos \\ Santos*, Rosiéli Martini**, Adenilde Salla****
}

\begin{abstract}
RESUMO: Foram estudadas 73 cepas de Staphylococcus aureus que apresentaram resistência à oxacilina e/ou cefoxitina na metodologia convencional e automação, isoladas no Hospital Universitário de Santa Maria no ano de 2008. O perfil de sensibilidade frente aos antimicrobianos foi avaliado através da difusão do disco e automação. Para o antimicrobiano vancomicina, foi realizada também a metodologia de microdiluição em caldo. Os isolados clínicos apresentaram resistência superior a $75 \%$ aos agentes $\beta$-lactâmicos, macrolídeos, aminoglicosídeos, lincosamidas e quinolonas e sensibilidade de $73,97 \%$ ao sulfametoxazol-trimetoprima. Houve $100 \%$ de sensibilidade à linezolida, daptomicina e vancomicina. Na deteç̧ão dos MRSA, o disco de oxacilina detectou um número maior de cepas resistentes comparado ao de cefoxitina. A automação teve desempenho equivalente ao disco de oxacilina. A associação de duas metodologias, automação e disco de oxacilina, pode constituir uma boa alternativa a ser adotada na rotina laboratorial.
\end{abstract}

Descritores: Staphylococcus aureus; Oxacilina; Cefoxitina.

ABSTRACT: We studied 73 strains of Staphylococcus aureus that presented resistance to oxacillin and cefoxitin in the conventional methodology and automation, isolated at the University Hospital of Santa Maria in 2008. The sensitivity profile to antimicrobials was valued by disc diffusion (DD) and automation $(\mathrm{Au})$. For the antibiotic vancomycin, the methodology of microdilution in broth was also done. The clinical isolates presented resistance superior to more than $75 \% \beta$-lactam agents, macrolides, aminoglycosides, lincosamides and quinolones and sensibility of $73.97 \%$ to trimethoprim-sulfamethoxazole. There was $100 \%$ of sensibility to linezolid, daptomycin, and vancomycin. In the detection of MRSA, the oxacillin disc detected a greater number of resistant strains compared to cefoxitin. The automation had performance equivalent to the oxacillin disc. The combination of both methodologies, automation and oxacillin disc, can be a good alternative to be adopted in routine laboratory.

Descriptors: Staphylococcus aureus; Oxacillin; Cefoxitin.

\footnotetext{
*Aluna do Curso de Graduação em Farmácia, Universidade Federal de Santa Maria, Santa Maria, Rio Grande do Sul, Brasil.

** Mestranda do Programa do Programa de Pós Graduação de Ciências Farmacêuticas, Universidade Federal de Santa Maria, Santa Maria, Rio Grande do Sul, Brasil.

*** Professora Adjunta do Departamento de Análises Clínicas e Toxicológicas, Universidade Federal de Santa Maria, Santa Maria, Rio Grande do Sul, Brasil.

${ }^{* * * *}$ Farmacêutica do Laboratório de Análises Clínicas do Hospital Universitário de Santa Maria - HUSM.
} 


\section{Introdução}

Staphylococcus aureus é um patógeno que se destaca por sua facilidade de propagação, principalmente no ambiente intra-hospitalar, relacionada à aquisição de resistência aos antimicrobianos. ${ }^{1}$ Normalmente, coloniza a pele, as fossas nasais e 0 intestino dos seres humanos. ${ }^{2}$ Sua importância clínica decorre da capacidade de causar vários tipos de infecções como foliculites, furúnculos, impetigo, até infecções sistêmicas potencialmente fatais. ${ }^{2}$

A partir da década de 60, o uso frequente das penicilinas penicilinase-estáveis, como a meticilina e a oxacilina, no tratamento das infecções estafilocócicas, favoreceu o surgimento de cepas resistentes a estes antimicrobianos, denominadas Staphylococcus aureus resistente à oxacilina/ meticilina (oxacillin-resistant Staphylococcus aureus - ORSA / methicillin-resistant Staphylococcus aureus - MRSA). ${ }^{3}$ Os ORSA/MRSA geralmente apresentam resistência estendida a todos os antimicrobianos $\beta$-lactâmicos ${ }^{4,5}$ e foram primeiramente reconhecidos como patógenos hospitalares. Entretanto, nos últimos anos, eles emergiram em infecções comunitárias. ${ }^{6}$

Dois tipos de resistência à oxacilina podem ser observados em S. aureus, estando envolvidos três mecanismos. A resistência clássica, ou intrínseca, é determinada pela presença do gene mecA localizado no cromossomo bacteriano. Esse gene é responsável pela síntese de proteínas ligadoras de penicilina (PBPs) alteradas, conhecidas como PBP2a ou PBP2', que ao substituírem outras PBPs na membrana acabam conferindo baixa afinidade tanto à oxacilina, quanto a outros antimicrobianos $\beta$-lactâmicos. ${ }^{4,5,7} \mathrm{O}$ outro tipo de resistência é a borderline, ou intermediária, é mais rara e ocorre quando cepas de $\mathrm{S}$. aureus que apresentam sensibilidade reduzida ou sensibilidade borderline à meticilina/oxacilina (Borderline Oxacillin-Resistant Staphylococcus aureus - BORSA) produzem uma grande quantidade de $\beta$-lactamases tipo A. ${ }^{8}$ Resistência borderline pode ocorrer também devido às modificações nas PBPs 1, 2 e 4 (Modified Penicillin-Binding Protein Staphylococcus aureus MODSA). ${ }^{8,9}$

As opções terapêuticas nas cepas com sensibilidade borderline são mais amplas, pois normalmente não existe resistência cruzada a outros $\beta$-lactâmicos ${ }^{8}$ (carbapenêmicos e cefalosporinas - com exceção das novas cefalosporinas as quais possuem atividade antiMRSA, que são a ceftarolina e ceftobiprole). Esta ausência de resistência cruzada é consenso para os MODSA; 8,9 porém para os BORSA ocorre divergência de interpretação, podendo ser considerada ausente ${ }^{8}$ e ou presente. ${ }^{9} \mathrm{~A}$ possibilidade de ocorrer múltipla resistência aos antimicrobianos não $\beta$-lactâmicos é rara para os BORSA e ausente para os MODSA. ${ }^{8,9} \mathrm{~A}$ vancomicina é a terapia de escolha para infecções graves causadas pelos MRSA, ${ }^{8}$ uma vez que a multirresistência dessas cepas limita as alternativas terapêuticas e exige um maior tempo de tratamento clínico. ${ }^{5}$ No entanto, já existem relatos de cepas que apresentaram suscetibilidade reduzida a este glicopeptídeo. ${ }^{11}$

Com o conhecimento desses três mecanismos associados a esse tipo de resistência, houve uma redefinição, sendo, desde 2010, definidas como MRSA/ORSA todas as cepas de S. aureus que expressam o gene mecA e outros mecanismos conhecidos como BORSA e MODSA que vão conferir resistência à oxacilina. ${ }^{13}$

O objetivo desse estudo foi avaliar as metodologias de difusão do disco com oxacilina e cefoxitina e sistema automatizado na detecção das cepas de S. aureus resistentes à meticilina, bem como o perfil de sensibilidade frente aos antimicrobianos, no Hospital Universitário de Santa Maria (HUSM). 


\section{Material e métodos}

Foram selecionadas, durante 0 ano de 2008, 73 cepas de S. aureus que apresentaram resistência à oxacilina e/ou cefoxitina pelo método de difusão do disco (DD) e/ou automação (Au) (MicroScan $®$ - Siemens) provenientes de diferentes isolados clínicos de pacientes atendidos ou internados no HUSM.

Avaliação da sensibilidade à oxacilina/cefoxitina: Teste da difusão do disco: Foi realizado de acordo com o Clinical and Laboratory Standards Institute (CLSI) $2008^{12}$ com os discos de oxacilina $(1 \mu \mathrm{g})$ e cefoxitina $(30 \mu \mathrm{g})$. Após 24 horas de incubação à $35^{\circ} \mathrm{C} \pm 2^{\circ} \mathrm{C}$, efetuou-se a leitura do diâmetro do halo de inibição do crescimento. Foram classificadas como sensíveis as amostras que apresentavam halos $\geq 13 \mathrm{~mm}$ para 0 disco de oxacilina e $\geq 22 \mathrm{~mm}$ para 0 de cefoxitina. Estas amostras foram classificadas como resistentes quando apresentavam halos de sensibilidade menores que os citados. ${ }^{12}$

As cepas ficaram armazenadas em caldo triptona de soja com $15 \%$ de glicerol a $-20^{\circ} \mathrm{C}$, até 0 ano de 2010, quando foram novamente ativadas. Seus perfis de sensibilidade foram reavaliados utilizando o novo painel lançado pela Siemens (Painel Combo gram-positivos - PC 29) do aparelho MicroScan, sistema autoSCAN®. Os resultados foram interpretados segundo os critérios estabelecidos pelo CLSI 2010.13

Devido ao fato de não ter ocorrido mudança nos critérios de interpretação entre o CLSI 2008 e - CLSI 2010, no que se refere aos diâmetros dos halos para os discos de oxacilina/cefoxitina, a análise comparativa das metodologias pode ser efetuada. ${ }^{12,13}$

A determinação da CIM para a vancomicina, no ano de 2010, foi realizada por metodologia automatizada, onde as concentrações desse antimicrobiano variaram de 32 a $0,25 \mu \mathrm{g} / \mathrm{mL}$ e pelo método convencional de determinação da concentração inibitória mínima (microdiluição em caldo), com concentrações de 256 a $0,25 \mu \mathrm{g} / \mathrm{mL}^{14}$ Os resultados obtidos nas duas metodologias foram interpretados conforme CLSI 2010.13

Para controle dos testes de sensibilidade foram utilizadas as cepas de S. aureus ATCC 29213, S. aureus ATCC 25923 e Enterococcus faecalis ATCC 51299.

O presente estudo foi aprovado pelo Comitê de Ética em Pesquisa da Universidade Federal de Santa Maria (UFSM), sob número 0117.0.243.000-08.

\section{Resultados}

Das 73 cepas estudadas, $18(24,6 \%)$ foram provenientes da Unidade de Terapia Intensiva (UTI) adulto, seguida do setor de pneumologia com 16/73 (21,9\%) e Pronto Atendimento (PA) adulto com 10/73 (13,7\%). As demais cepas, 29/73 (39,8\%), foram isoladas de outras unidades hospitalares.

Em relação ao espécime clínico, observou-se que os maiores isolamentos $17 / 73$ cepas $(23,3 \%)$ provieram de secreção traqueal, seguida de escarro e secreções em geral com 14/73 $(19,2 \%)$ cada. Foram incluídas como secreções em geral: secreções de escara, de membros inferiores, de gastrostomia e de ferida operatória.

Nas Tabelas 1 e 2 encontram-se os perfis de sensibilidade das cepas frente aos antimicrobianos utilizando automação e difusão do disco, respectivamente. Os antimicrobianos listados foram classificados de acordo com o CLSI $2010^{12}$ nos grupos: A (primeira escolha, testados e reportados na Saúde (Santa Maria), v.37, n.1, p. 23-30,

rotina); B (primeira escolha, testados e reportados seletivamente, agentes importantes Sousa, L. U.; Mielke, T, P.; Horner, R.; Rodrigues, M. A.; Santos, S. O.; Martini, R.; Salla, A. 
principalmente em infecções nosocomiais); C (suplementares e reportados seletivamente, agentes alternativos quando ocorrem cepas resistentes a várias drogas primárias); $O$ (outros não rotineiramente utilizados) e Inv. (em investigação).

Tabela 1 - Perfil de sensibilidade frente aos antimicrobianos das 73 cepas de S. aureus utilizando automação (MicroScan® - Siemens) de acordo com os breakpoints do CLSI 2010.

\begin{tabular}{|c|c|c|c|c|}
\hline Antimicrobiano $\left(^{*}\right)$ & $\begin{array}{c}\text { Sensível } \\
n(\%)\end{array}$ & $\begin{array}{c}\text { Intermediário } \\
n(\%)\end{array}$ & $\begin{array}{c}\text { Resistente } \\
\mathrm{n}(\%)\end{array}$ & $\begin{array}{l}\text { Total } \\
\mathrm{n}(\%)\end{array}$ \\
\hline Ampicilina (0) & - & - & $73(100)$ & $73(100)$ \\
\hline Penicilina (A) & - & - & $73(100)$ & $73(100)$ \\
\hline Oxacilina (A) & $8(10,96)$ & - & $65(89,04)$ & $73(100)$ \\
\hline Amoxicilina - ácido clavulânico (0) & $9(12,33)$ & - & $64(87,67)$ & $73(100)$ \\
\hline Ceftriaxona $(0)$ & $9(12,33)$ & - & $64(87,67)$ & $73(100)$ \\
\hline Meropenem (0) & $9(12,33)$ & - & $64(87,67)$ & $73(100)$ \\
\hline Ampicilina - Sulbactam (0) & $10(13,70)$ & - & $63(86,30)$ & $73(100)$ \\
\hline Cefazolina $(0)$ & $10(13,70)$ & - & $63(86,30)$ & $73(100)$ \\
\hline Imipenem (0) & $10(13,70)$ & - & $63(86,30)$ & $73(100)$ \\
\hline Clindamicina (A) & $15(20,55)$ & - & $58(79,45)$ & $73(100)$ \\
\hline Eritromicina $(A)$ & $14(19,18)$ & $1(1,37)$ & $58(79,45)$ & $73(100)$ \\
\hline Gentamicina $(C)$ & $16(21,92)$ & $1(1,37)$ & $56(76,71)$ & $73(100)$ \\
\hline Levofloxacina (C) & $16(21,92)$ & $1(1,37)$ & $56(76,71)$ & $73(100)$ \\
\hline Rifampicina (B) & $40(54,79)$ & $1(1,37)$ & $32(43,84)$ & $73(100)$ \\
\hline Sulfametoxazol - Trimetoprima (A) & $54(73,97)$ & - & $19(26,03)$ & $73(100)$ \\
\hline Daptomicina (B) & $73(100)$ & - & - & $73(100)$ \\
\hline Linezolida (B) & $73(100)$ & - & - & $73(100)$ \\
\hline Vancomicina (B) & $73(100)$ & - & - & $73(100)$ \\
\hline
\end{tabular}

$\left.{ }^{*}\right)$ Grupo a que pertence o antimicrobiano segundo o CLSI 2010

Tabela 2 - Perfil de sensibilidade frente aos antimicrobianos das cepas de S. aureus utilizando a metodologia de difusão do disco de acordo com os breakpoints do CLSI 2008.

\begin{tabular}{|c|c|c|c|c|}
\hline Antimicrobiano(*) & $\begin{array}{c}\text { Sensível } \\
n(\%)\end{array}$ & $\begin{array}{c}\text { Intermediário } \\
\mathrm{n}(\%)\end{array}$ & $\begin{array}{c}\text { Resistente } \\
\text { n (\%) }\end{array}$ & Total (\%) \\
\hline Oxacilicina (A) & $3(4,11)$ & $1(1,37)$ & $69(94,52)$ & $73(100)$ \\
\hline Cefoxitina $^{a}$ & $8(10,95)$ & - & $65(89,05)$ & $73(100)$ \\
\hline Azitromicina $(\mathrm{A})$ & $10(14,08)$ & - & $61(85,92)$ & $71(100)$ \\
\hline Clindamicina (A) & $12(16,44)$ & - & $61(83,56)$ & $73(100)$ \\
\hline Teicoplanina (Inv.) & $64(96,97)$ & - & $2(3,03)$ & $66(100)$ \\
\hline Tigeciclinab & $71(98,61)$ & - & $1(1,39)$ & $72(100)$ \\
\hline Vancomicina (B) & $65(100)$ & - & - & $65(100)$ \\
\hline
\end{tabular}

Saúde (Santa Maria), v.37, n.1, p. 23-30,

a Cefoxitina é agente antimicrobiano para predição de resistência dos estafilococos mediada pelo gene mecA. 0 teste com disco de cefoxitina tem a finalidade de detectar a resistência à oxacilina e não à própria cefoxitina. Conforme o diâmetro deve-se relatar como resistente ou sensível à oxacilina. (CLSI, 2008; Mimica, 2007$){ }^{12}$ b $O$ antimicrobiano tigeciclina não possui halo na listagem do CLSI (2008), entretanto foi aprovado pelo Food and Drug Administration (FDA) em junho de 2005, o qual definiu seus breakpoints. ${ }^{15}$ 
A partir dos resultados dos perfis de sensibilidade obtidos através das metodologias de $\mathrm{DD}$ e Au, determinamos diferentes perfis ao associar e comparar as metodologias (Tabela 3).

Tabela 3 - Perfis de sensibilidade frente à cefoxitina e oxacilina das 73 cepas de S. aureus pelas metodologias de difusão do disco e automação.

\begin{tabular}{c|c|c|c|c}
\hline \multirow{2}{*}{ Perfil } & & & Au & Total (\%) \\
\hline I & OXA & CFO & OXA & \\
\hline II & R & R & R & $73(100)$ \\
\hline III & R & R & S & $73(100)$ \\
\hline IV & R & S & R & $71(100)$ \\
\hline V & S & S & R & $73(100)$ \\
\hline VI & R & S & S & $66(100)$ \\
\hline VII & I & R & S & $72(100)$ \\
\hline
\end{tabular}

S = Sensível; I = Intermediário; R = Resistente; NT = Não Testado

Na Tabela 4, encontram-se as frequências das CIM para a vancomicina das 73 cepas do nosso estudo.

Tabela 4 - Frequência das Concentrações Inibitórias Mínimas para vancomicina nas 73 cepas de S. aureus pela Microdiluição em Caldo e pela Automação (MicroScan® - Siemens).

\begin{tabular}{c|c|c}
\hline CIM & MDC & Au \\
& $n(\%)$ & $n(\%)$ \\
\hline $0,25 \mu \mathrm{g} / \mathrm{mL}(\mathrm{S})$ & $4(5,5 \%)$ & - \\
\hline $0,5 \mu \mathrm{g} / \mathrm{mL}(\mathrm{S})$ & $11(15,1 \%)$ & - \\
\hline $1 \mu \mathrm{g} / \mathrm{mL}(\mathrm{S})$ & $43(58,9 \%)$ & $52(71,2)$ \\
\hline $2 \mu \mathrm{g} / \mathrm{mL}(\mathrm{S})$ & $15(20,5 \%)$ & $21(28,8)$ \\
\hline
\end{tabular}

\section{Discussão}

S. aureus meticilina resistente (MRSA) tornou-se o maior problema clínico e epidemiológico na última década, ${ }^{16}$ estando envolvido em infecções nosocomiais e comunitárias encontrandose espalhado em todo o mundo. ${ }^{17}$ Este fato levou ao uso em excesso dos glicopeptídios, especialmente a vancomicina e, com isso, surgiram cepas de $\mathrm{S}$. aureus resistentes à vancomicina (Vancomycin-resistant S. aureus- VRSA). ${ }^{18}$ Por essa razão, é importante utilizar na rotina uma metodologia rápida, mas com acurácia na detecção dos MRSA.

A UTI adulto foi a unidade hospitalar que mais contribuiu para os isolados de MRSA do nosso estudo, com 24,6\% (18/73), seguido do setor de pneumologia com 21,9\% (16/73) e 0 PA adulto com 13,7\% (10/73).

A aquisição de infecção por MRSA caracteriza um evento importante, pois aumenta a morbimortalidade dos pacientes e a prevalência desse microrganismo em UTI é variável. ${ }^{19,20}$ Um estudo realizado em um hospital de São Paulo, em 2007, encontrou uma taxa de $70 \%$ de MRSA em UTI. ${ }^{21}$ Já em 2008, em um laboratório de Uruguaiana (RS), foram isolados $18 \%$ de Saúde (Santa Maria), v.37, n.1, p. 23-30, 2011.

Sousa, L. U.; Mielke, T, P.; Horner, R.; Rodrigues, M. A.; Santos, S. O.; Martini, R.; Salla, A. MRSA em UTI. ${ }^{22}$ 
O uso prévio de antimicrobianos é um fator de risco para o desenvolvimento de pneumonia por microrganismos potencialmente resistentes, como os MRSA, ${ }^{23}$ os quais têm emergido como um dos principais agentes causadores desta patologia. ${ }^{19} \mathrm{Em}$ nossa pesquisa, secreção traqueal e escarro foram os espécimes clínicos onde houve o maior percentual de isolamento de cepas MRSA, totalizando $42,5 \%$.

A resistência a múltiplas classes de antimicrobianos tem sido uma característica das cepas de MRSA hospitalares, o que pode levar a falha terapêutica em virtude da dificuldade de se encontrar a antibioticoterapia ideal. . $^{16,24,25}$

Nas Tabelas 1 e 2, podemos observar que, além dos altos índices de resistência aos agentes $\beta$-lactâmicos, houve resistência a outras classes de antimicrobianos como: macrolídeos, aminoglicosídeos, lincosamidas e quinolonas, apresentando taxas de resistência superiores a $75 \%$. Frente à levofloxacina, apenas $21,92 \%$ das cepas foram sensíveis (Tabela 1), dado esse que está de acordo com um estudo anterior, realizado no mesmo nosocômio, por Tizzotti et. al., em 2010, onde foi encontrada uma taxa de sensibilidade frente à levofloxacina de $36,7 \%$ em cepas $\mathrm{S}$. aureus resistente à oxacilina. ${ }^{26}$ Esse índice encontrado é preocupante pela relevância que este antimicrobiano tem na terapia de infecções do trato respiratório.

Por outro lado, as cepas do nosso estudo apresentaram sensibilidade de 73,97\% (54/73) ao sulfametoxazol-trimetoprima (Tabela 1). Resultado semelhante foi encontrado por Vilela, em 2004, no Hospital Universitário Oswaldo Cruz, em Pernambuco, onde a sensibilidade ao sulfametoxazol-trimetoprima nas cepas de MRSA estudadas foi de 73,6\%. ${ }^{27}$ Além disso, também verificamos significativa sensibilidade aos antimicrobianos teicoplanina $(96,97 \%)$, tigeciclina $(98,61 \%)$. Todas as cepas foram sensíveis à daptomicina, linezolida e vancomicina $(100 \%)$.

Avaliando-se os perfis de desempenho dos discos de oxacilina e cefoxitina e da oxacilina no método automatizado (Tabela 3 ), verificou-se que a maioria dos isolados clínicos estudados (79,5\%-58/73) foi resistente nos três testes efetuados para a detecção das cepas MRSA (perfil I). Três cepas foram detectadas somente pela automação, isto é, sensíveis aos discos de oxacilina e cefoxitina, e duas foram detectadas apenas pelo disco de oxacilina. Portanto, neste estudo a automação apresentou desempenho equivalente ao disco de oxacilina.

Apesar do CLSI recomendar o uso da cefoxitina para predizer a resistência à oxacilina em S. aureus ${ }^{13}$ no presente estudo a cefoxitina não se mostrou superior à oxacilina ( $D D$ e $\left.A u\right)$. A partir de 2004, o CLSI tem recomendado que o disco de cefoxitina deve ser preferido ao de oxacilina na detecção da resistência mediada pelo gene mecA, pelo fato dele possuir maior capacidade de indução na expressão da PBP2a do que a oxacilina. ${ }^{7,16,28}$ Swenson e colaboradores, em 2005, encontraram desempenho equivalente com os discos de cefoxitina, oxacilina e microdiluição em caldo com oxacilina. ${ }^{29}$ Kaiser e colaboradores, em 2010, em um estudo realizado em um hospital de Vitória (ES), obtiveram 100\% de especificidade e sensibilidade com DD de oxacilina e cefoxitina e CIM com o teste de diluição em ágar; no sistema automatizado (Microscan Walkaway), a sensibilidade foi de $92,9 \%$ e $85 \%$ de especificidade. ${ }^{25}$

O CLSI, em 2009, excluiu o teste de DD para vancomicina para S. aureus, pois ele não a de metodologias para a detecção de cepas de Staphylococcus aureus resistentes à meticilina (MRSA) e análise do perfil de sensibilidade frente aos antimicrobianos em um hospital terciário diferencia os isolados sensiveis dos intermediários, passando a preconizar apenas a MDC para a determinação da CIM para a vancomicina. ${ }^{30} \mathrm{~A}$ sensibilidade a vancomicina nas cepas 
estudadas, foi de $100 \%$ na Au e MDC. De acordo com a Tabela 4 verificamos que a CIM mais frequente nos dois métodos foi de $1 \mu \mathrm{g} / \mathrm{mL}$ e que 73 (100\%) das cepas apresentaram resultados iguais em ambos os métodos. No entanto, é de conhecimento que as técnicas microdilucionais apresentam erro intrínseco de \pm 1 diluição. ${ }^{31}$

\section{Considerações finais}

A escolha da melhor metodologia fenotípica para a detecção dos MRSA permanece controversa. ${ }^{16}$ Neste estudo, o disco de oxacilina foi superior ao disco de cefoxitina na detecção da resistência à oxacilina em $\mathrm{S}$. aureus. Embora os resultados obtidos pela difusão do disco com oxacilina e pela automação tenham sido bastante concordantes, o uso associado desses dois métodos corrobora para uma melhor deteç̧ão dessa resistência.

\section{Referências}

1. Enright MC, Robinson DA, Randle G, Feil EJ, Grundmann H, Spratt BG. The evolutionary history of methicillinresistant Staphylococcus aureus (MRSA).PNAS 2002; 99(11): 7687-98.

2. Koneman EW, Stephen AD, Janda WM, Schreckenberger PC, Winn WC. Diagnóstico Microbiológico. Texto e Atlas Colorido. $6^{a}$ ed. Rio de Janeiro: Guanabara-Koogan; 2008.

3. Hiramatsu K, Katayama Y, Yuzawa H, Ito T. Molecular genetics of methicillin-resistant Staphylococcus aureus. Int J Med Microbiol 2002; 292: 67-74.

4. Lowy FD. Antimicrobial resistance: the example of Staphylococcus aureus. J Clin Invest 2003; 111(9): 1265-73.

5. Santos AL, Santos DO, Freitas CC, Ferreira BLA, Afonso IF, Rodrigues CR, et al. Staphylococcus aureus: visitando uma cepa de importância hospitalar. J Bras Patol Med Lab 2007; 43(6): 413-23.

6. Weber JT. Community-Associated Methicillin-Resistant Staphylococcus aureus. Clin Infect Dis 2005; 41(4): 269-72.

7. Chambers HF. Methicillin resistance in Staphylococci: molecular and biochemical basis and clinical implications. Clin Microbiol Rev 1997; 10(4): 781-91.

8. Rossi F, Andreazzi DB. Resistência bacteriana: Interpretando o antibiograma. São Paulo: Atheneu, 2005.

9. Organização Pan-Americana de Saúde. Boas Práticas em Microbiologia Clínica, Agência Nacional de Vigilância Sanitária, Coordenação Geral de Laboratórios de Saúde Pública - CGLAB/SVS/MS, Laboratório Central do Hospital São Paulo da Universidade Federal de São Paulo. São Paulo (SP), 2008.

10. Khorvash F, Mostafavizadeh K, Mobasherizadeh S. Frequency of mecA gene and borderline oxacillin resistant Staphylococcus aureus in nosocomial acquired methicillin resistance Staphylococcus aureus infections. Pak J Biol Sci 2008; 11(9): 1282-85.

11. Hiramatsu K, Aritaka N, Hanaki H, Kawasaki S, Hosoda Y, Hori S, et al. Dissemination in Japanese hospitals of strains of Staphylococcus aureus heterogeneously resistant to vancomycin. The Lancet 1997; 35: 1670-3.

12. Clinical and Laboratory Standards Institute (CLSI). Performance standards for antimicrobial susceptibility testing; eighteenth informational supplement, document M100-S18. Wayne, PA, USA: CLSI, 2008.

13. Clinical and Laboratory Standards Institute (CLSI). Performance standards for antimicrobial susceptibility testing, twentieth information supplement, document M100-S20. Wayne, PA, USA: CLSI, 2010.

Saúde (Santa Maria), v.37, n.1, p. 23-30, 2011.

Sousa, L. U.; Mielke, T, P.; Horner, R.; Rodrigues, M. A.; Santos, S. O.; Martini, R.; Salla, A.

14. Clinical and Laboratory Standards Institute (CLSI). Methods for dilution antimicrobial susceptibility tests for bacteria that grow aerobically; approved standard, seventh edition, document M7-A7. Wayne, PA, USA: CLSI, 2006. 
15. Food and Drug Administration - FDA - Disponível em: < http://www.accessdata.fda.gov/drugsatfda_docs/label/2010/021821s021/bl.pdf >. Acesso em: 23.11.2010.

16. Mimica MJ, Berezin EN, Carvalho RLB, Mimica IM, Mimica LMJ, Sáfadi MAP, et al. Detection of Methicillin Resistance in Staphylococcus aureus Isolated from Pediatric Patients: Is the Cefoxitin Disk Diffusion Test Accurate Enough? Braz J Infect Dis 2007; 11(4): 415-17.

17. Chambers H.F. The changing epidemiology of Staphylococcus aureus? Emerg Infect Dis 2001; 7: 178-82.

18. Centers for Disease Control and Prevention (CDC). Staphylococcus aureus resistant to vancomycin - United States, 2002. Morb Mortal Wkly Rep 2002; 51: 565-7.

19. Kollef M. SmartApproaches for Reducing Nosocomial Infections in the ICU. Chest 2008; 134: 447-56.

20. Gelatti LC, Becker AP, Bonamigo RR, d'Azevedo PA. Staphylococcus aureus resistentes à meticilina: disseminação emergente na comunidade. An Bras Dermatol 2009; 84(5): 501-6.

21. Moreira M, Freitas MR, Martins ST, Castelo A, Medeiros EAS. Efficacy of a Program of Prevention and Control for Methicillin-Resistant Staphylococcus aureus Infection in an Intensive-Care Unit. Braz J Infect Dis 2007; 1(1): $57-62$.

22. Klein G, Goularte LS. Prevalência de Staphylococcus aureus multirresistentes em amostras biológicas do Laboratório Osvaldo Cruz, Uruguaiana-RS.Rev Bras Farm 2008; 89(2): 121-4.

23. Froes F, Paiva JA, Amaro P, Baptista JP, Brum G, Bento H, et al. Documento de consenso sobre pneumonia nosocomial. Rev Port Pneumol 2007; 13(3):419-86.

24. Cuevas O, Cercenado E, Vindel A, Guinea J, Sanchez-Conde M, Sanchez-Somolinos M, et al. Evolution of the Antimicrobial Resistance of Staphylococcus spp. in Spain: Five Nationwide Prevalence Studies. Antimicrobial Agent Chemother 2004; 48(11): 4240-5.

25. Kaiser TDL, Pacheco FC, Lima AA, Pereira EM, Santos KRN, Nunes APF. Avaliação de métodos comumente usados em laboratórios para a determinação da suscetibilidade à oxacilina entre amostras de Staphylococcus sp, isoladas de um hospital de Vitória, Estado do Espírito Santo. Rev Soc Bras Med Trop 2010; 43(3): 298-303.

26. Tizzotti MK, Horner R, Kempfer CB, Martini R, Mayer LE, Roehrs M, et al. Prevalência e perfil de sensibilidade de Staphylococcus aureus isolados em um Hospital Escola na cidade de Santa Maria, Brasil. Revista Saúde 2010; 36(1): 47-56.

27. Vilela MA. Padrão de resistência antimicrobiana de casos de infecções nosocomiais no Recife, Pernambuco, Brasil, 2002-2003. 91 f. Dissertação (Mestrado em Saúde Pública) - Fundação Oswaldo Cruz, Recife, 2004.

28. Sharp SE, Warren JA, Thomson RB. Cefoxitin disk diffusion screen for confirmation of oxacillin-resistant Staphylococcus aureus isolates and utility in the clinical laboratory. Diagn Microbiol Infect Dis 2005; 51: 69-71.

29. Sweenson JM, Tenover FC and the Cefoxitin Disk Study Group. Results of Disk Diffusion Testing with Cefoxitin Correlate with Presence of mecA in Staphylococcus spp. J Clin Microbiol 2005; 43(8): 3818-23.

30. Clinical and Laboratory Standards Institute (CLSI). Performance standards for antimicrobial susceptibility testing; nineteenth informational supplement, document M100-S19. Wayne, PA, USA: CLSI, 2009.

31. Swenson JM, Anderson KF, Lonsway DR, Thompson A, McAllister SK, Limbago BM, et al. Accuracy of Commercial and Reference Susceptibility Testing Methods for Detecting Vancomycin-Intermediate Staphylococcus aureus. J Clin Microbiol 2009; 47(7): 2013-17.

\section{Rosmari Hörner}

Endereço para correspondência - Departamento de Análises Clínicas e Toxicológicas - Universidade

Saúde (Santa Maria), v.37, n.1, p. 23-30,

2011.

metodologias para a detecção de cepas de Staphylococcus aureus resistentes à meticilina (MRSA) e análise do perfil de sensibilidade frente aos antimicrobianos em um hospital terciário
Federal de Santa Maria. Prédio 26, Sala 1201, Campus UFSM, CEP: 97105-900. Santa Maria, RS.

E-mail: rosmari.ufsm@gmail.com

Recebido em 04 de julho de 2011.

Aprovado em 02 de setembro de 2011 